nature

\title{
UK's wasted opportunity at UNCSTD
}

Whatever may or may not be decided at UNCSTD in Vienna these two weeks, it is perfectly clear that the world scientific and technological community is experiencing strong pressures for change, for instance through proposals for the establishment of a massive global research and development fund, and through demands that the legal framework for the transfer of technology be restructured. It is thus vitally important that all those who attend UNCSTD take back to their home country a clear impression of the forces at work and alert their colleagues in every possible way to the need for new thinking. To a degree press reports help, but a very important ingredient is what individual people who attend do.

The constitution of the British delegation to UNCSTD is
- for whatever reason - such as to make this job very difficult to perform for the scientific and technological community. A delegation of twenty or so could have included some well connected scientists, some influential industrialists, some representatives of education, and some non-governmental development experts in addition to the necessary complement of civil servants. Instead the delegation comprises a ininister (for two days), eight civil servants, five representatives of the Vienna embassy, three private or personal secretaries and only two people who could in any sense be regarded as outsiders. The burden on these two to inform the broadest community is intolerable. It will be interesting to see what help they get from their civil-servant colleagues in the months ahead.

\section{Science, nonsense and responsibility}

THE right - and responsibility - of the layman to exert an influence on the actions of scientists and technologists is becoming firmly established, as is evidenced this week by the controversy over the exclusion of journalists from an international meeting on short-term testing for carcinogens (see p.623). But is there not an equal right and responsibility for the scientist to guide the layman? Guidance is surely needed. The misuse of science by those often lacking in scientific training and understanding, but never lacking either conviction or commercial acumen, is now commonplace. It is commonplace because there are profits to be made from it.

For example, it is there even in the bookshops. A new form of escapism has been packaged as an alternative to science fiction, romantic fiction and thrillers. The new genre treats as fact a wide range of spurious topics. Themes include the artefacts left by our extragalactic forefathers, the possibility of disappearing through triangular holes in the sky over the Caribbean, the everyday lives of UFOflying folk, paranormal fork-bending and outrageous claims about the influence that this additive-free food or that celestial body might have on our lives. A factor common to all these books, it seems, is the need for belief. A recent example of the extent to which strongly held but totally unfounded belief can resist the weight of fact is the saga of the alleged anti-cancer drug laetrile.

Can this need for belief be satisfied by something more sober than UFOs and cancer cures? By, say, a belief that an analytical and scientific approach to a physical problem will lead to as near as we can get to a solution? This will be possible only if genuine critical science, with its clarities and uncertainties, is sold to the public with the determination that sells pseudo-science. It does not help to hold closed meetings and so increase the public's natural distrust of 'the expert'. The exercise is difficult, but it must be undertaken, or the voice of reason will not be heard at all.

True science does answer back sometimes. The BBC Horizon series carried an excellent critique of von Daniken's books. But many thousands more of his books will have been sold since then. Science should answer back more loudly and more often. One medium which invites such a response is the relatively new one (in the UK) of local radio, which thrives on the cheap-to-run 'phone-in' programme. In these programmes all forms of fringe and suspect forms of pseudo-science are often discussed by 'experts', and the more far-fetched the thesis proposed the more enthusiastic the response from the listeners. Just as harmful are 'open-line' programmes in which callers attempt to discuss subjects which neither the caller nor the programme presenter comprehends. Presenters are generally conscientious and, especially on medical matters, make pleas for calls from more qualified listeners. The pleas are often unanswered. This seems a pity because, although discussion of this type are certain to be shallow and are often not conducted very well, a shallow discussion with some sense in it must be better than one with no sense.

It may be true that 'truth will out', as it did eventually for laetrile. But scientific truth will 'out' much faster if it is sold with more enthusiasm, and if this means descending to the level of the market place, then perhaps that's as it should be. 